Bundesgesundheitsbl 2013 $\cdot 56: 584-584$

DOI 10.1007/s00103-013-1680-y

○) Springer-Verlag Berlin Heidelberg 2013

Mitteilungen des Arbeitskreises Blut des Bundesministeriums für Gesundheit

\title{
Sicherheit von Blut und Blutprodukten angesichts aktueller Berichte über die Übertragbarkeit der Alzheimer-Krankheit im Tierexperiment
}

\section{Stellungnahme}

\section{Bei der 73. Sitzung des Arbeitskreises Blut am 27.06.2012 wurde folgende Stellungnahme verabschiedet (S11):}

Es gibt zunehmend Hinweise, dass die Proteinablagerungen im Hirngewebe, die als mögliche Ursache der Alzheimer-Erkrankung angesehen werden, durch eine Veränderung von körpereigenen Eiweißmolekülen entstehen. Diese als Umfaltung bezeichnete Veränderung kann durch zunächst wenige umgefaltete und zusammengelagerte Eiweißmoleküle angeregt werden, die dann als „Saatkeim“ wirken und so die Umfaltung weiterer Eiweißmoleküle bewirken. Dieser Mechanismus findet sich auch bei einigen anderen Erkrankungen, u. a. der Creutzfeldt-JakobKrankheit und der infolge des Verzehrs von Fleischprodukten BSE-kranker Rinder aufgetretenen Variante dieser Erkrankung.

Da diese Variante der CreutzfeldtJakob-Krankheit in seltenen Fällen (4 Fälle in Großbritannien) durch eine Bluttransfusion übertragen wurde, kam die Frage auf, ob auch die Alzheimer-Erkrankung so übertragen werden könnte. Für viel Aufmerksamkeit sorgten daher Ergebnisse einer US-amerikanischen Arbeitsgruppe, die seit 2011 auf verschiedenen Fachtagungen vorgestellt wurden. Die Forscher hatten Mäusen ein menschliches Gen übertragen, das eine Veranlagung zur Alzheimer-Krankheit bedingt. Diese Tiere entwickeln mit der Zeit Alzheimer-typische Eiweißablage- rungen. Eine Übertragung von Blut von alten in jüngere Mäuse führte dazu, dass bei den jüngeren Tieren diese Veränderungen früher einsetzten, als dies ohne Transfusion der Fall war. Allerdings wurde durch die Blutübertragung nicht das Vollbild der Alzheimer-ähnlichen Krankheit ausgelöst. Bislang wurden diese Forschungsergebnisse weder in wissenschaftlich nachprüfbarer Weise ausführlich veröffentlicht, noch von anderen Labors bestätigt.

Für den Menschen gibt es bislang auch keinen epidemiologischen Hinweis, der auf einen solchen Zusammenhang hindeutet.

Ohne Zweifel geben die experimentellen Daten jedoch Anlass zu weiterer Forschung. Die Sicherheit von Blut und Blutprodukten ist nach derzeitigem Kenntnisstand nicht infrage gestellt.

\section{Fazit}

Aus den bisher vorliegenden experimentellen Daten in Tiermodellen kann eine Übertragbarkeit der Alzheimer-Erkrankung von Mensch zu Mensch nicht abgeleitet werden.

Weitere Erläuterungen finden Sie in der wissenschaftlichen Ergänzung zur Stellungnahme.

Für den Arbeitskreis Blut

Prof. Dr. R. Burger, Vorsitzender

Dr. R. Offergeld, Geschäftsführerin 